\title{
Worst-Case Access Delay of HomePlug Green PHY (HPGP) for Delay-Critical In-Vehicle Applications
}

\author{
Zhengguo Sheng ${ }^{1}$, Mümin Özpolat ${ }^{2}$, Daxin Tian $^{3}$, Victor Leung ${ }^{4}$, and Maziar Nekovee ${ }^{1}$ \\ ${ }^{1}$ Department of Engineering and Design, University of Sussex, UK \\ ${ }^{2}$ WMG, University of Warwick, UK \\ ${ }^{3}$ School of Transportation Science and Engineering, Beihang University, China \\ ${ }^{4}$ Department of Electrical and Computer Engineering, University of British Columbia, Canada \\ Email: z.sheng@sussex.ac.uk
}

\begin{abstract}
The increasing complexity of automotive electronics has put considerable pressure on automotive communication networking to accommodate in-vehicle information flows. The use of power lines has been a promising alternative to in-vehicle communications because of elimination of extra data cables. In this paper, we focus on the latest HomePlug Green PHY (HPGP) which has been promoted by major automotive manufacturers for green communications with electric vehicles, and study its worst-case access delay performance in supporting delaycritical in-vehicle applications using both theoretical analysis and the simulation. Specifically, we apply Network Calculus as a deterministic modeling approach to evaluate the worst delay and further verify its performance using the OMNeT++ simulation. Evaluation results are also supplemented to compare with legacy methods and provide useful guidelines for developing HPGP based vehicular power line communication systems.

Index Terms-Vehicular power line communications, delay analysis, Network Calculus, OMNeT++.
\end{abstract}

\section{INTRODUCTION}

With the emerging automated tasks in vehicle domain, the development of in-vehicle communications is increasingly important and subjected to new applications [1]. Although both wired and wireless communications have been largely used for supporting diverse applications, most of in-vehicle applications with delay-critical nature, such as brake and engine controls, still prefer dedicated wired networks for reliable transmission. According to Ford Motor Company, "today's vehicles have more than 2,000 wires, which would measure more than a mile in length" [2]. The weight of a wire harness is in the region of 20 to 50 kilograms per car, which makes up the third heaviest and costliest component in a car, right behind the chassis and engine.

The legacy in-vehicle communication buses, such as controller area network (CAN) [3] and local interconnect network (LIN) [4], are highly application specific and usually interconnected in a heterogeneous network via gateways. To cope with the increasing bandwidth demand of future applications, a number of recent studies addressed the performance of Ethernet with IEEE 802.1 AVB [5], [6] as in-vehicle network

This research was sponsored by The Engineering, and Physical Sciences Research Council (EPSRC) (EP/P025862/1), Royal Society-Newton Mobility Grant (IE160920) and Asa Briggs Visiting Fellowship from University of Sussex. to deliver multimedia services. The on-going development of AVB towards time-sensitive networking (TSN) [7] will further extend its capability to support stringent real-time applications. These applications of different network technologies and point-to-point links, however, lead to an inflexible network architecture and a complex cable harness in vehicles. In order to cope with next generation vehicle intelligence, vehicle manufactures are always on the lookout for cost effective approaches in order to reduce complexity, weight and cost of material as well as achieve fuel efficiency.

Over the past few years, we have witnessed an increasing interest in the use of power line communication (PLC) for home automation systems, automatic meter reading, real-time energy management systems, and many other applications. The use of power line communications (PLC) is promising and novel to in-vehicle applications. Latest research efforts have so far been focused on the study of innovative Physical and MAC protocol design [8], [9]. However, industry PLC solutions have been developing for more than a decade. The latest HomePlug Green PHY (HPGP) standard has been promoted by major automotive manufacturers as the common communication interface to facilitate the integration of electric vehicles into future smart grid applications. Therefore, it is of great interest to find out whether the HomePlug solution can support invehicle applications, particularly, a fundamental understanding of HPGP limitations in supporting delay-critical in-vehicle traffic flows with various priorities and delay requirements.

In this paper, we focus on the access delay ${ }^{1}$ which is the major bottleneck of contention based transmission, and employ a deterministic modeling approach, namely Network Calculus (NC) [10], to characterize the worst delay performance of realtime in-vehicle transmission based on HPGP. NC has been recently developed as a powerful tool to model and analyze congestion and access controls of switch Ethernet and cellular networks [11], [12]. However, there is no such a work on the analysis of contention based access protocol for in-vehicle environment. Moreover, we build an in-vehicle PLC network simulator using OMNeT++ which is compliant to the HPGP

\footnotetext{
${ }^{1}$ It represents the interval between the time when a frame reaches the head-of-line and the beginning of the successful transmission. To simplify the notation, we also use "delay" to represent access delay in the paper.
} 


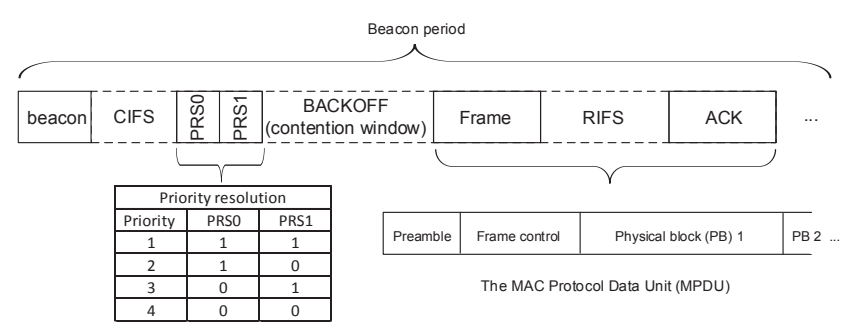

Fig. 1. HomePlug Green PHY CSMA/CA Medium Access

specification [13] to verify our findings. The obtained results can provide useful guidelines in determining the optimal frame length and scheduling strategy to maximum the bandwidth efficiency of HPGP in vehicle environment.

The remainder of this paper is organized as follows. In section II, we characterize the HPGP MAC protocol, in-vehicle traffic requirements and data modeling. Section III provides the analytical result of delay performance using NC tool. The performance evaluation supplemented by both numerical results and simulation are provided in Section IV, and the paper is concluded in Section V.

\section{In-Vehicle Data Modeling}

\section{A. HomePlug GP MAC scheduling}

HPGP uses Carrier Sense Multiple Access (CSMA) as the basic channel access mechanism and it supports four different channel access priorities to provide differentiated Quality of Service (QoS) for latency-sensitive applications, which is accomplished during the priority resolution period as shown in Fig. 1. The cycle length of HPGP (or beacon period) is defined as $C_{\mathrm{BP}}=40 \mathrm{~ms}$, which is preconfigured by the central coordinator $(\mathrm{CCo})$ to match a cycle frequency of $50 \mathrm{~Hz}$ based on the network time base. Let $i \in\{1,2, \ldots, N\}$ denote the priority level of flows with $N$ corresponding to the lowest priority. The HPGP defines 4 priority levels and thus we have $N=4$. The transmission frequency of a priority flow $i$ can be defined as $F_{i}=f(i) \cdot C_{\mathrm{BP}}$, which means that a new transmission can be scheduled every $F_{i}$ time. The $f(i)$ is the cycle rate of the priority flow $i$. For example, if we consider a case that a higher priority flow needs to be transmitted more frequently, we may have $f(i)=i$. Since all flows are sent in a cyclic manner, the least common multiple $(\mathrm{lcm})$ of transmission frequencies of all priority flows is $F=\operatorname{lcm}\left\{F_{1}, F_{2}, \ldots, F_{N}\right\}$. It is noted that the cycle time of in-vehicle traffic can be varied from $10 \mathrm{~ms}$ up to $500 \mathrm{~ms}$ depending on the applications [14]. The traffic pattern of HPGP of all priority levels can be well suited within the allowed service rate of control traffic class [15].

\section{B. In-vehicle traffic characteristics and data modeling}

The general in-vehicle traffic is categorized in Table I, which is supported by several coexisting in-vehicle communication buses. For example, the LIN bus which is primarily used in the body and comfort domains supports 8 byte data length
TABLE I

DATA TRAFFIC REQUIREMENTS FOR DELAY-CRITICAL IN-VEHICLE APPLICATIONS

\begin{tabular}{|l|l|l|}
\hline Traffic class & Max delay & Data rate \\
\hline Control \& Management & $10 \mathrm{~ms}[14]$ & $20 \mathrm{Kbps}-1 \mathrm{Mbps}$ \\
\hline Safety data (audio) & $33 \mathrm{~ms}[16]$ & $64 \mathrm{Kbps}-1.4 \mathrm{Mbps}$ \\
\hline Infotainment data & $150 \mathrm{~ms}[17]$ & $\sim 1.5 \mathrm{Mbps}$ \\
\hline
\end{tabular}

with low safety requirement, whereas the CAN bus which is used in powertrain and driver assistant control domains can support up to 8 byte data length but with stringent delay and transmission rate requirements. Given a typical invehicle frame length of up to 8 byte, we consider the same case [18] that the short MAC protocol Data Unit (MPDU) with only frame control (128 bits), which is defined in IEEE 1901, can be employed by HPGP for in-vehicle communications purposes. The total transmission time for such a frame under the Mini-ROBO ${ }^{2}$ transmission rate $3.8 \mathrm{Mbps}$ is $658.08 \mu \mathrm{s}$, which includes 2 priority resolution slots $(35.84 \mu \mathrm{s}$ per slot), an average of 3.5 backoff slots ( $35.84 \mu$ s per slot), one control frame $(110.48 \mu \mathrm{s})$, one response interfame space (RIFS) $(140 \mu \mathrm{s})$, one acknowledgement $(110.48 \mu \mathrm{s})$ and one contention interframe space (CIFS) $(100 \mu \mathrm{s})$. Therefore, to simplify our analysis, we can use an equivalent frame length of short MPDU $L_{s}=2500$ bits including the aforementioned protocol overhead and data frame.

\section{AcCess Delay AnALysis}

\section{A. Arrival curve}

We start with the analysis of collision free transmissions in which collisions can be solved by priority resolution or backoff counter. The actual HPGP media access is performed cyclic. Successful transmission depends on both priority and contention of each flow. According to the definition in [10], the arrival flow of a message type with priority $i$ is a cumulative function and can be derived as the step function $A_{i}(t)=\left\lceil\frac{t}{F_{i}}\right\rceil$. $L_{i}$. The cumulative arrivals with priority higher than $i$ can be derived as

$$
A_{i}^{h}(t)=\sum_{i=1}^{i-1} A_{i}(t)=\sum_{i=1}^{i-1}\left\lceil\frac{t}{F_{i}}\right\rceil \cdot L_{i},
$$

In order to ease the analysis of the deterministic performance of the network, the upper bounded arrival curve (1) can be characterized by the well known token bucket controller concept which can be defined as $\alpha_{i}(t)=\sigma_{i}+\rho_{i} \cdot t$, where $\sigma$ is the maximum amount of flow that can arrive in a burst and $\rho$ is the average rate of the flow. Additionally, the data arrival rate is limited by the capacity of link which is denoted as $R$. Thus the arrival curve can be defined as

$$
\alpha_{i}(t)=\min \left\{R \cdot t, \sigma_{i}+\rho_{i} \cdot t\right\}
$$

\footnotetext{
${ }^{2}$ In this paper, we only consider the Mini-ROBO model for the sake of the worst delay performance. The obtained results can be applied to other models, such as standard and high speed ROBO.
} 
In essence, the arrival curve for a particular priority flow can be characterized by $\left(R, \sigma_{i}, \rho_{i}\right)$. In our case, the burst of each priority flow can be assumed as $\sigma_{i}=L_{i}$, which means that an immediate transmission can happen at $t=0$. The average rate of a flow can be defined as $\rho_{i}=\frac{L_{i}}{F_{i}}$. Consequently, the linear expression of cumulative arrivals with priority higher than $i$ can be obtained as

$$
\alpha_{i}^{h}(t)=\min \left\{R \cdot t, \sigma_{i}^{h}+\rho_{i}^{h} \cdot t\right\} .
$$

where $\sigma_{i}^{h}=(i-1) \cdot L_{i}$ and $\rho_{i}^{h}=\sum_{j=1}^{i-1} \frac{L_{j}}{F_{j}}$. Thus we can easily observe that $\alpha_{i}^{h}(t) \geq A_{i}^{h}(t)$ when $t \geq 675 \mu \mathrm{s}^{3}$, which is negligible compared to $C_{\mathrm{BP}}$ and a long term observation. It is worth noting that the token bucket model has been widely used in packet-switched Internet networks to define conformant flows, thus the derived upper arrival curve satisfies all constraints of an affine arrival curve in network calculus.

\section{B. Service curve}

The outgoing flow, which is served by the communication channel, can be modeled by a family of simple service curve called the rate-latency service curve $\beta(t)=R \cdot(t-T)^{+}$. Consider the priorities of individual flow and the non-preemptive nature of transmission mechanism, we derive the service curves for each priority traffic as follows.

1) Highest priority: For the highest priority traffic, it can always grant channel access unless there is lower priority frame on transmission. Thus the rate-latency curve is

$$
\beta_{1}(t)=R_{1} \cdot\left(t-T_{1}\right)^{+} .
$$

where $R_{1}=R$ and $T_{1}=\frac{\max \left\{L_{i} \mid i \in 2 \ldots N\right\}}{R}$ which is the maximum transmission latency of a low priority data. For example, if we consider to assign $L_{s}$ for all priority flows, then $T_{1}=658.08 \mu \mathrm{s}$.

2) middle priority: The difference between a lower priority flow and the highest one is that any lower priority flow needs to wait until all higher priority flows are served. So there is an additional latency to process the initial burst imposed from higher priority flows. Moreover, according to the aggregate traffic modeling of non-preemptive priority flows [19], the equivalent service rate is limited to $R-\sum_{j=1}^{i-1} \rho_{j}$. The service curve is derived as

$$
\beta_{i}(t)=R_{i} \cdot\left(t-T_{i}\right)^{+} .
$$

where $R_{i}=R-\sum_{j=1}^{i-1} \rho_{j}$ and $T_{i}=\frac{\sum_{j=1}^{i-1} \sigma_{j}}{R_{i}}+T_{1}$. The results can be applied to characterize second and third priority flows in HPGP.

3) Lowest priority: The only latency imposed for this flow is the waiting time to serve all higher flows, so we can derive the service curve for the lowest priority flow as

$$
\beta_{N}(t)=R_{N} \cdot\left(t-T_{N}\right)^{+} .
$$

where $R_{N}=R-\sum_{i=1}^{N-1} \rho_{i}$ and $T_{N}=\frac{\sum_{i=1}^{N-1} \sigma_{i}}{R_{N}}$.

\footnotetext{
${ }^{3}$ The result can be easily derived by comparing the step function (1) with the linear approximation (3).
}

\section{Delay bound}

Theorem 1: The upper delay bound $d_{i}$ for each priority flow in HPGP is

$$
T_{1} \leq d_{i} \leq T_{i}+\frac{\sigma_{i} \cdot\left(R-R_{i}\right)}{\left(R-\rho_{i}\right) \cdot R_{i}} .
$$

Proof: According to the definition in [10], the delay bound of a flow $i$ is the maximum horizontal deviation between its arrival curve $\alpha_{i}$ and service curve $\beta_{i}$, which can be expressed as follows.

$$
\begin{gathered}
d_{i} \leq \sup _{t \geq 0}\left\{\inf \left\{\tau \geq 0: \alpha_{i}(t) \leq \beta_{i}(t+\tau)\right\}\right\} \\
\Rightarrow d_{i} \leq \sup _{t \geq 0}\left\{\inf _{\tau \geq 0:}\left\{\min \left\{R \cdot t, \sigma_{i}+\rho_{i} \cdot t\right\}=R_{i} \cdot\left(t+\tau-T_{i}\right)^{+}\right\}\right\},
\end{gathered}
$$

According to min-plus algebra, the distributivity of sup and inf with respect to operators $\vee(\max )$ and $\wedge$ (min) can lead (8) into

$d_{i} \leq \sup _{t \geq 0}\left\{\inf _{\tau \geq 0:}\left\{R \cdot t=\beta_{i}(t+\tau)\right\} \wedge \inf _{\tau \geq 0:}\left\{\sigma_{i}+\rho_{i} \cdot t=\beta_{i}(t+\tau)\right\}\right.$,

Since we know that when $t \leq \frac{\sigma_{i}}{R-\rho_{i}}, R \cdot t \leq \sigma_{i}+\rho_{i} \cdot t$. By denoting $t_{i}^{*}=\frac{\sigma_{i}}{R-\rho_{i}}$ which is the curving point of arrival curve, we can further derive (9) as

$$
\begin{aligned}
d_{i} \leq \quad & \sup _{0 \leq t \leq t_{i}^{*}}\left\{\inf _{\tau \geq 0:}\left\{R \cdot t=R_{i} \cdot\left(t+\tau-T_{i}\right)^{+}\right\}\right\} \\
& \vee \sup _{t \geq t_{i}^{*}}\left\{\inf _{\tau \geq 0:}\left\{\sigma_{i}+\rho_{i} \cdot t=R_{i} \cdot\left(t+\tau-T_{i}\right)^{+}\right\}\right\},
\end{aligned}
$$

For $t \geq T_{i}-\tau,(10)$ leads to

$$
\begin{aligned}
d_{i} \leq \quad & \sup _{0 \leq t \leq t_{i}^{*}}\left\{\inf _{\tau \geq 0:}\left\{\tau=\frac{\sum_{i=1}^{i-1} \rho_{i} \cdot t}{R_{i}}+T_{i}\right\}\right\} \\
& \vee \sup _{t \geq t_{i}^{*}}\left\{\inf _{\tau \geq 0:}\left\{\tau=\frac{\sigma_{i}+\left(\sum_{i=1}^{i} \rho_{i}-R\right) \cdot t}{R_{i}}+T_{i}\right\}\right\} .
\end{aligned}
$$

Due to the linear increasing and decreasing of inner equations with respect to $t$ in (11), the result is obtained when $t=t_{i}^{*}$. $\square$

According to Theorem 1, the delay performance is deteriorated with decreasing of priority level. Therefore, the worst delay performance can be expected when the lowest priority flow is applied for transmission and thus the maximum transmission delay by using HPGP for in-vehicle transmission is $d_{N}$. According to (7), we can derive the maximum delay as

$$
d_{4} \leq T_{4}+\frac{\sigma_{4} \cdot\left(R-R_{4}\right)}{\left(R-\rho_{4}\right) \cdot R_{4}}=\frac{3 \cdot L_{s}}{R-\rho_{i}^{h}}+\frac{L_{s} \cdot \rho_{i}^{h}}{\left(R-\rho_{i}\right)\left(R-\rho_{i}^{h}\right)} .
$$

where $\rho_{i}^{h}=\sum_{j=1}^{3} \frac{L_{s}}{F_{i}}$. Assuming that all priority flows are with the highest frequency of transmission $F_{1}=F_{2}=F_{3}=$ $F_{4}=C_{\mathrm{BP}}$, we can obtain the worst delay performance of HPGP as $2.1 \mathrm{~ms}$ which can satisfy delay requirement of major control and safety application classes defined by CAN and LIN buses [14], [15], e.g., $10 \mathrm{~ms}$. 
The total bandwidth utility of HPGP can be derived as

$$
U_{B W}=\frac{\sum_{i=1}^{N} \rho_{i}}{R}=\frac{0.25 \mathrm{Mbps}}{3.8 \mathrm{Mbps}}=6.5 \% \text {. }
$$

The result shows that the bandwidth utility is quite low and there is a significant potential to better utilize HPGP to cope with bandwidth demanding applications. In the following, we will find out the maximum frame length (equivalently, the maximum transmission rate) that can be supported by HomePlug GP without violating the delay requirement.

Result 1: Given a hard delay deadline of a delay-critical in-vehicle application $d_{\max }$, the maximum frame length that can be supported by HomePlug GP is defined in (14), and the transmission rate of each priority flow can be obtained as

$$
\rho_{i}=R-\frac{L_{i} \cdot \rho_{i}^{h}}{\left(d_{i}^{\max }-T_{1}^{*}\right) \cdot\left(R-\rho_{i}^{h}\right)-\sum_{j=1}^{i-1} L_{j}} .
$$

where $T_{1}^{*}=T_{1}$ if $i \neq N$; else, $T_{1}^{*}=0$, and $\rho_{i}^{h}=\sum_{j=1}^{i-1} \rho_{j}$.

Proof: According to (12), the lowest priority flow experiences the longest transmission delay. Therefore, in order to ensure a hard delay requirement in priority based access channel, a transmission should be considered with lowest priority in the worst scenario. Since the worst delay performance is when $F_{1}=F_{2}=F_{3}=F_{4}=C_{\mathrm{BP}}$, we can obtain the following

$$
\begin{aligned}
& d_{\max }=\frac{3 x C_{\mathrm{BP}}}{R-3 x}+\frac{3 x^{2} C_{\mathrm{BP}}}{(R-x)(R-3 x)} \\
& \Rightarrow 3 d_{\max } x^{2}-\left(4 R d_{\max }+3 C_{\mathrm{BP}} R\right) x+R^{2} d_{\max }=0 \\
& \Rightarrow x=\frac{4 R d+3 C R \pm \sqrt{4 R^{2} d^{2}+9 C^{2} R^{2}+24 R^{2} d C}}{6 d} .
\end{aligned}
$$

where $x=L_{\max } / C_{\mathrm{BP}}, C=C_{\mathrm{BP}}$ and $d=d_{\max }$. Since the maximum total transmission rate cannot excess the channel capacity, that is, $\frac{4 L_{\max }}{C_{\mathrm{BP}}} \leq R$, the maximum frame length (14) can be obtained. Then (15) can be obtained from (7).

Result 1 is useful in the sense that a maximum number of Physical Block (PB) can be allocated for one Physical Protocol Data Unit (PPDU). Recall from Section II that the $L_{s}=2500$ is the equivalent protocol overhead and the short MPDU (preamble and frame control), the maximum number of PBs that can be supported by the in-vehicle HomePlug GP is

$$
N_{\mathrm{PB}}=\left\lfloor\frac{L_{\max }-2500}{136 \cdot 8}\right\rfloor \text {. }
$$

where 136 is the number of bytes of a PB defined by MiniROBO. In priority based multi-access transmission, delay and transmission rate are two important criteria to evaluate the effectiveness of transmission scheduling. Hence, we propose a delay guaranteed maximum rate scheduling algorithm to maximize the bandwidth utilization and maintain delay deadline by imposing the fairness feature into consideration.

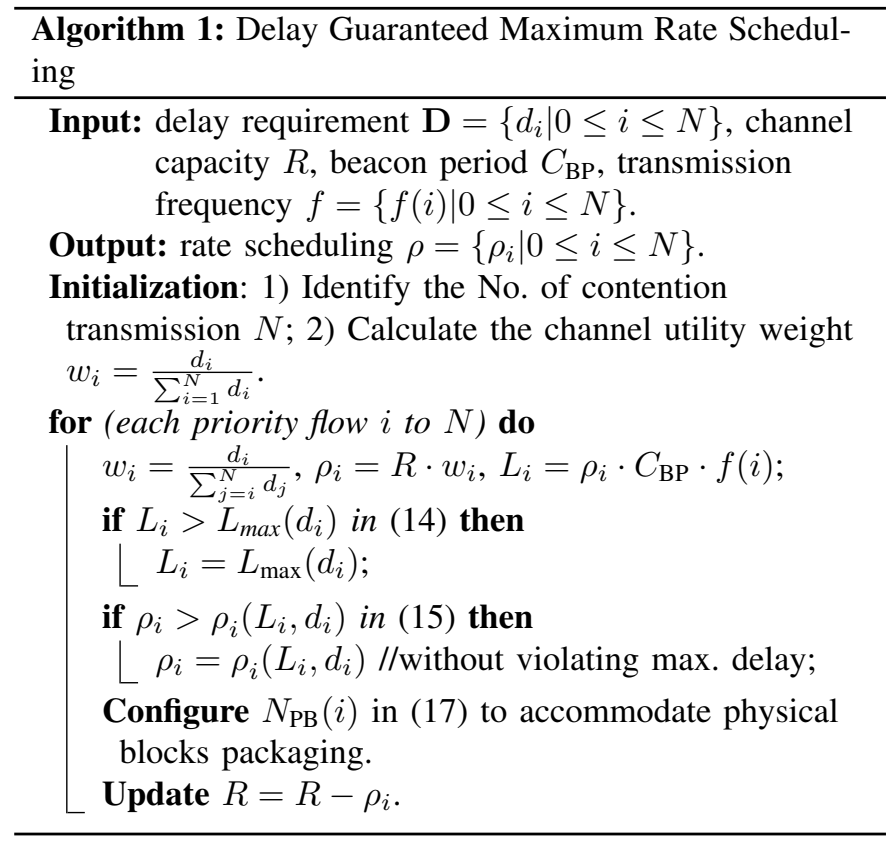

\section{Collision impact on delay performance}

So far we have shown that the delay performance of HPGP among different priorities is positive to support in-vehicle traffic in collision free scenarios. However, when the number of nodes increases on a sharing bus, it is highly possible that more than one nodes will share the same priority and thus collision may happen. Fig. 2 shows the collision probability in terms of the number of contending nodes on the same priority and the size of Contention Window (CW).

Therefore, the maximum delay performance can be characterized as follows

$d_{\mathrm{worst}}= \begin{cases}2.1 \mathrm{~ms}, & \text { collision free } \\ \mathrm{BPC} \cdot C_{\mathrm{BP}}+\max _{\mathrm{i}=1 \ldots 4}\left\{d_{i}(\mathrm{BPC}, \mathrm{CW})\right\}, & \text { collision }\end{cases}$

where BPC is the Backoff Procedure Event Counter. The value of $\mathrm{BPC}, \mathrm{CW}$, priority and the corresponding delay performance which can be calculated according to Theorem 1 are listed in the Table II. It is clear that when collision happens, i.e., BPC $>=1$, the worst delay performance cannot satisfy the hard requirement of in-vehicle control and safety applications, which is largely because of the beacon periods incurred by the retransmission. For the cases when more nodes need to access the channel, our work [20] and [18] have proposed modified HPGP solutions to achieve collision free transmission for in-vehicle networks, which is out of scope of this paper. Compared with Table I, the worst delay bounds obtained show that it is not recommended to use HPGP in a direct manner for delay-critical in-vehicle communications, particularly when the number of nodes and priorities are not properly configured to avoid collision.

\section{PERformance Evaluation}

In this section, we provide both numerical and simulation results. 


$$
L_{\max }=\min \left\{\frac{4 d_{\max } R C_{\mathrm{BP}}+3 C_{\mathrm{BP}}^{2} R-\sqrt{\left(2 d_{\max } R C_{\mathrm{BP}}+3 R C_{\mathrm{BP}}^{2}\right)^{2}+12 d_{\max } R^{2} C_{\mathrm{BP}}^{3}}}{6 d_{\max }}, \frac{R C_{\mathrm{BP}}}{4}\right\} .
$$

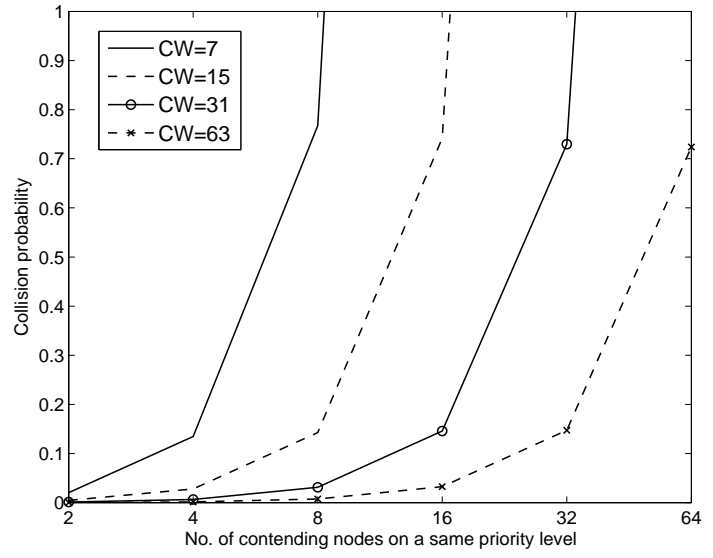

Fig. 2. Collision probability under different node number (CW: 7, 15, 31, 63 are the $\mathrm{CW}$ size defined by HPGP).

TABLE II

THE WORST DELAY PERFORMANCE AS A FUNCTION OF CONTENTION WINDOW (CW) SIZE, BACKOFF PROCEDURE EVENT COUNTER (BPC) AND PRIORITY

\begin{tabular}{|c|c|c|c|}
\cline { 2 - 4 } \multicolumn{1}{c|}{} & Priorities & Priorities & \multicolumn{1}{c|}{} \\
$1 \& 2$ & $3 \& 4$ & $d_{\text {worst }}$ \\
\hline $\mathrm{BPC}=0$ & $\mathrm{CW}=7$ & $\mathrm{CW}=7$ & $2.1 \mathrm{~ms}$ \\
\hline $\mathrm{BPC}=1$ & $\mathrm{CW}=15$ & $\mathrm{CW}=15$ & $42.6 \mathrm{~ms}$ \\
\hline $\mathrm{BPC}=2$ & $\mathrm{CW}=15$ & $\mathrm{CW}=31$ & $83.7 \mathrm{~ms}$ \\
\hline $\mathrm{BPC}>2$ & $\mathrm{CW}=31$ & $\mathrm{CW}=63$ & $40 \cdot \mathrm{BPC}+5.9 \mathrm{~ms}$ \\
\hline
\end{tabular}

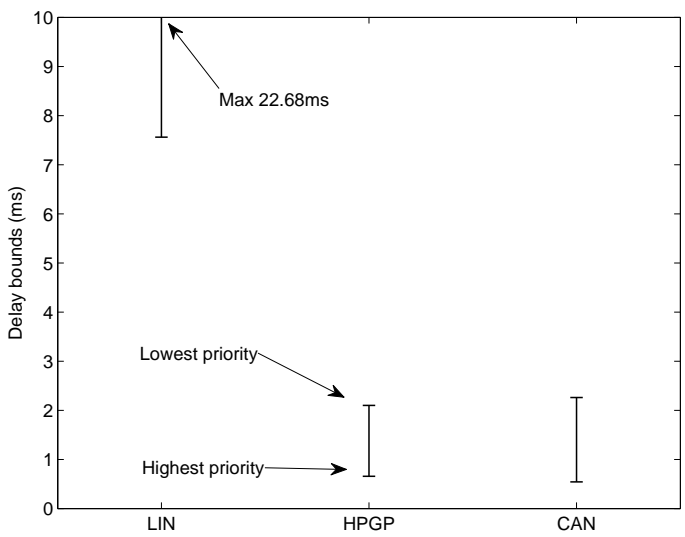

Fig. 3. Delay bounds comparisons

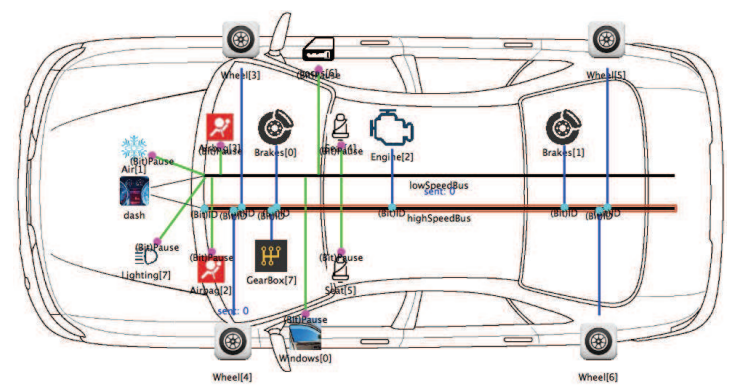

Fig. 4. Simulation topology

\section{A. Numerical result}

Fig. 3 shows the delay bounds ranging from the highest to lowest priority flows among four major solutions obtained using NC. In the comparison, we assume the identical data size of 8 byte and 4 priorities for each solution. According to the standard specification, the LIN bus [4] uses the master-slave periodical transmission with date rate of $20 \mathrm{Kbps}$, whereas the CAN [3] bus uses priority-based contention detection and resolution (CDR) with date rate of $250 \mathrm{Kbps}$. It shows that the HPGP has clear advantage over the LIN, but with competitive performance to the CAN based protocols.

\section{B. Simulation results}

We adopt an in-vehicle communication network from a real car service manual and reproduce it into Fig. 4 as the simulation topology, where a number of nodes are connected via buses to the dashboard (or CCo). The communication bus is the HPGP based PLC, but can also be replaced by CAN or LIN, etc. We build an in-vehicle PLC network simulator using OMNeT++ which is compliant to the HPGP specification [13]. In the simulation, we assume each HPGP node can send messages at a random transmission rate but has to follow $F_{i}=i \cdot C_{\mathrm{BP}}$, where $i=1 \ldots N$. The priority level of each node $i$ is fixed and distinct from other nodes in one simulation in order to avoid collision, but can be randomly changed in another one. The results in Fig. 5 and 6 are averaged over 500 independent simulations. To further compare its delay performance with legacy solutions, we also develop the source code $^{4}$ of LIN and CAN in the same real-time OMNeT++ environment. To create a realistic setting, we set the clock cycle timing based on the CAN specification [3] and LIN specification [4], respectively.

Fig. 5 shows the average delay performance of a node in a 4-node scenario using the standard HPGP. It is clear that the HPGP performance is much better than the LIN and closed to the CAN. Moreover, the simulation is consistent with the

\footnotetext{
${ }^{4}$ Our simulation code is available at: https://github.com/Mioja/DelayComparision-of-HomePlug-GP-CAN-M-CAN-and-LIN
} 


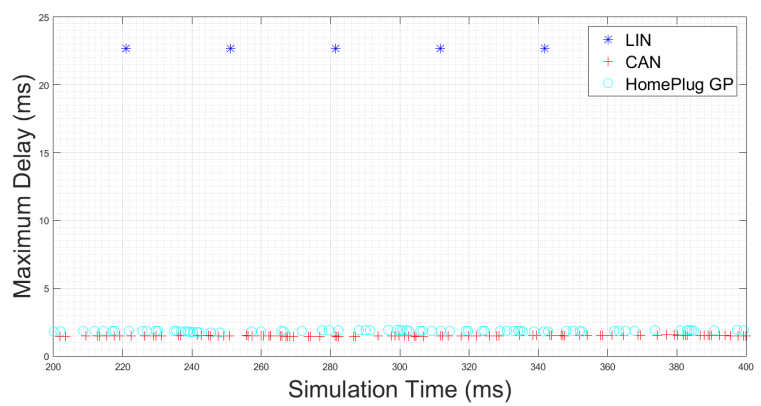

Fig. 5. Simulation result of the 4 nodes scenario

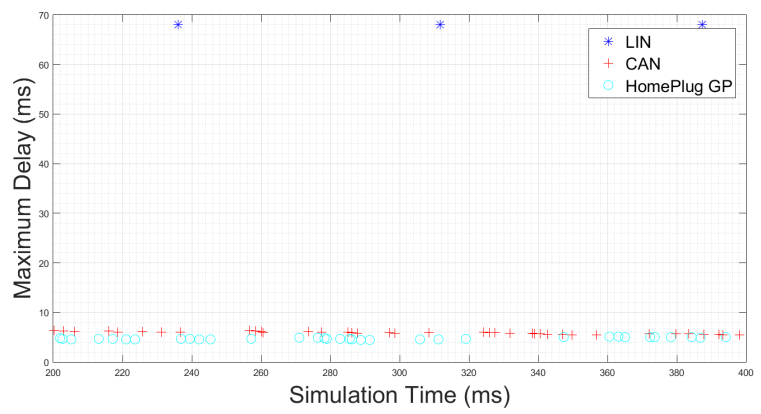

Fig. 6. Simulation result of the 10 nodes scenario

Theorem 1 and theoretical result in Fig. 3. In Fig. 6, the modified HPGP [18] is adopted to cope with more nodes, i.e., 10 nodes, the HPGP based solution performs even better than the standard CAN and thus can better cope with a large node scenarios. It is worth noting that the number of contending nodes over a single bus in vehicle should be properly designed in order to satisfy the hard delay requirement. In practice, a hierarchical or star topology is usually adopted to reduce the contention.

\section{Proposed fair scheduling method}

Table III shows the results of the scheduled transmission rate and achievable delay performance by applying Algorithm 1. In this example, we simply configure $F_{1}=F_{2}=F_{3}=$ $F_{4}=C_{\mathrm{BP}}$. As can be seen, the derived result can keep the actual delay performance within the targeted delay deadline. Moreover, more adaptive transmission rates (by adjusting the number of PBs) can be scheduled for all flows in order to maximize the bandwidth utility. The fairness index indicates that the proposed solution can successfully maintain a global fairness to allocate more bandwidth to lower priority flows.

TABLE III

A RESULT OF THE PROPOSED SCHEDULING METHOD

\begin{tabular}{|l|l|l|l|l|}
\hline Priority level & 1 & 2 & 3 & 4 \\
\hline Targeted delay (ms) & 7 & 12 & 40 & 50 \\
\hline Achieved delay (ms) & 6.8 & 9 & 14.3 & 36.8 \\
\hline Transmission rate (Mbps) & 0.18 & 0.42 & 1.39 & 1.74 \\
\hline Maximum physical blocks & 4 & 8 & 19 & 21 \\
\hline Bandwidth utility & $98.2 \%$ & \multicolumn{4}{|l|}{$(3.73 \mathrm{Mbps} / 3.8 \mathrm{Mbps})$} \\
\hline
\end{tabular}

\section{Conclusions}

In this paper, we have analyzed the access delay performance of HPGP based in-vehicle PLC and fairly compared its performance with other legacy solutions. Through the theoretical analysis and simulation results, we have proven that HPGP is able to meet the hard delay requirement of invehicle communications, given a careful design of network topology. Our results indicate that the HPGP is promising to replace the existing LIN or CAN bus networks and to support delay-critical in-vehicle applications.

\section{REFERENCES}

[1] W. Fleming, "Forty-year review of automotive electronics: A unique source of historical information on automotive electronics," IEEE Veh. Tech. Mag., vol. 10, no. 3, pp. 80-90, Sept 2015.

[2] Ford Motor Company. "Improving Vehicle Connectivity". Accessed: 19 October 2016. [Online]. Available: http://bit.ly/1ZusLlA

[3] "CAN specification version 2.0", in Robert Bosch GmbH, 1991.

[4] "LIN specification package revision 2.1," in LIN Consortium, 2006.

[5] M. Johas Teener, A. Fredette, C. Boiger, P. Klein, C. Gunther, D. Olsen, and K. Stanton, "Heterogeneous networks for audio and video: Using ieee 802.1 audio video bridging," Proc. of the IEEE, vol. 101, 2013.

[6] L. Bello, "Novel trends in automotive networks: A perspective on ethernet and the ieee audio video bridging," in Proc. IEEE Emerging Technology and Factory Automation (ETFA), Sept 2014, pp. 1-8.

[7] S. Kehrer, O. Kleineberg, and D. Heffernan, "A comparison of faulttolerance concepts for ieee 802.1 time sensitive networks (tsn)," in Proc. IEEE Emerging Technology and Factory Automation (ETFA), Sept 2014.

[8] N. Taherinejad, L. Lampe, and S. Mirabbasi, "Adaptive impedance matching for vehicular power line communication systems," in Proc. Intl. Symp. on Power Line Comm. and its Apps. (ISPLC), 2014, p. 214.

[9] Z. Sheng, A. Kenarsarii, N. Taherinejad, and V. Leung, "A multichannel medium access control protocol for vehicular power line communication systems," IEEE Trans. on Veh. Tech., vol. 65, no. 2, 2016.

[10] J.-Y. Le Boudec and P. Thiran, Network Calculus: A Theory of Deterministic Queuing Systems for the Internet. Springer-Verlag, 2001.

[11] M. Fidler, "Survey of deterministic and stochastic service curve models in the network calculus," IEEE Commu. Surv. Tut., vol. 12, 2010.

[12] J. Huang, Y. Sun, Z. Xiong, Q. Duan, Y. Zhao, X. Cao, and W. Wang, "Modeling and analysis on access control for device-to-device communications in cellular network: A network-calculus-based approach," IEEE Trans. on Veh. Tech., vol. 65, no. 3, pp. 1615-1626, March 2016.

[13] "HomePlug Green PHY specification release revision 1.1.1," in HomePlug Alliance, 2013.

[14] U. Klehmet, T. Herpel, K. Hielscher, and R. German, "Delay bounds for can communication in automotive applications," in Proc. MMB, 2008.

[15] S. Tuohy, M. Glavin, C. Hughes, E. Jones, M. Trivedi, and L. Kilmartin, "Intra-vehicle networks: A review," IEEE Trans. Intel. Trans. Sys., 2015.

[16] M. Rahmani, R. Steffen, K. Tappayuthpijarn, E. Steinbach, and G. Giordano, "Performance analysis of different network topologies for invehicle audio and video communication," in Proc. Intl. Telecom. Netw. Workshop on QoS in Multiservice IP Networks, Feb 2008, pp. 179-184.

[17] L. C. Wolf, C. Griwodz, and R. Steinmetz, "Multimedia communication," Proc. of the IEEE, vol. 85, no. 12, pp. 1915-1933, 1997.

[18] T. Gehrsitz, H. Kellermann, H.-T. Lim, and W. Kellerer, "Analysis of medium access protocols for power line communication realizing in-car networks," in Proc. IEEE Veh. Tech. Conf. (VTC Fall), Sept 2014, pp. $1-7$.

[19] J.-P. Georges, T. Divoux, and E. Rondeau, "Strict priority versus weighted fair queueing in switched ethernet networks for time critical applications," in IEEE Intl. Paral. and Dist. Proc. Symp., 2005, pp. 141141.

[20] R. Antonioli, M. Roff, Z. Sheng, J. Liu, and V. Leung, "A real-time mac protocol for in-vehicle power line communications based on homeplug gp," in Proc. IEEE Veh. Tech. Conf. (VTC Spring), 2015, pp. 1-5. 